\title{
The Michigan Spine Surgery Improvement Collaborative: a statewide Collaborative Quality Initiative
}

\author{
Victor Chang, MD, 1,4 Jason M. Schwalb, MD, 1,2,4 David R. Nerenz, PhD,, ${ }^{1,2,4}$ Lisa Pietrantoni, BS, ${ }^{1,4}$ \\ Sharon Jones, ${ }^{1,4}$ Michelle Jankowski, MAS, ${ }^{3,4}$ Nancy Oja-Tebbe, BS, ${ }^{3,4}$ \\ Stephen Bartol, MD, MBA, FRCSC, ${ }^{1,4,5}$ and Muwaffak Abdulhak, MD, FRCSC ${ }^{1,4}$
}

Departments of ${ }^{1}$ Neurosurgery, ${ }^{3}$ Public Health Sciences, and ${ }^{5}$ Orthopedics; ${ }^{2}$ Center for Health Policy and Health Services Research; and ${ }^{4} \mathrm{MSSIC}$ Coordinating Center, Henry Ford Hospital, Detroit, Michigan

OBJECT Given the scrutiny of spine surgery by policy makers, spine surgeons are motivated to demonstrate and improve outcomes, by determining which patients will and will not benefit from surgery, and to reduce costs, often by reducing complications. Insurers are similarly motivated. In 2013, Blue Cross Blue Shield of Michigan (BCBSM) and Blue Care Network (BCN) established the Michigan Spine Surgery Improvement Collaborative (MSSIC) as a Collaborative Quality Initiative (CQI). MSSIC is one of the newest of 21 other CQIs that have significantly improved-and continue to improve - the quality of patient care throughout the state of Michigan.

METHODS MSSIC focuses on lumbar and cervical spine surgery, specifically indications such as stenosis, disk herniation, and degenerative disease. Surgery for tumors, traumatic fractures, deformity, scoliosis, and acute spinal cord injury are currently not within the scope of MSSIC. Starting in 2014, MSSIC consisted of 7 hospitals and in 2015 included another 15 hospitals, for a total of 22 hospitals statewide. A standardized data set is obtained by data abstractors, who are funded by BCBSM/BCN. Variables of interest include indications for surgery, baseline patient-reported outcome measures, and medical history. These are obtained within 30 days of surgery. Outcome instruments used include the EQ-5D general health state score ( 0 being worst and 100 being the best health one can imagine) and EQ-5D-3 L. For patients undergoing lumbar surgery, a 0 to 10 numeric rating scale for leg and back pain and the Oswestry Disability Index for back pain are collected. For patients undergoing cervical surgery, a 0 to 10 numeric rating scale for arm and neck pain, Neck Disability Index, and the modified Japanese Orthopaedic Association score are collected. Surgical details, postoperative hospital course, and patient-reported outcome measures are collected at 90-day, 1-year, and 2-year intervals.

RESULTS As of July 1, 2015, a total of 6397 cases have been entered into the registry. This number reflects 4824 eligible cases with confirmed surgery dates. Of these 4824 eligible cases, 3338 cases went beyond the 120-day window and were considered eligible for the extraction of surgical details, 90 -day outcomes, and adverse events. Among these 3338 patients, there are a total of 2469 lumbar cases, 862 cervical cases, and 7 combined procedures that were entered into the registry.

CONCLUSIONS In addition to functioning as a registry, MSSIC is also meant to be a platform for quality improvement with the potential for future initiatives and best practices to be implemented statewide in order to improve quality and lower costs. With its current rate of recruitment and expansion, MSSIC will provide a robust platform as a regional prospective registry. Its unique funding model, which is supported by BCBSM/BCN, will help ensure its longevity and viability, as has been observed in other CQIs that have been active for several years.

http://thejns.org/doi/abs/10.3171/2015.10.FOCUS15370

KEY WORDS spine registry; quality improvement; prospective database

ABBREVIATIONS BCBSM = Blue Cross Blue Shield of Michigan; $B C N=$ Blue Care Network; $C Q I=$ Collaborative Quality Initiative; DVT = deep venous thrombosis; FTE = full-time employee; IRB = institutional review board; MCID = minimum clinically important difference; $\mathrm{mJOA}=$ modified Japanese Orthopaedic Association; MSSIC = Michigan Spine Surgery Improvement Collaborative; MUSIC = Michigan Urological Surgery Improvement Collaborative; $\mathrm{N}^{2} \mathrm{QOD}=$ National Neurosurgery Quality and Outcomes Database; NDI = neck disability index; NRS = numeric rating scale; $\mathrm{ODI}=$ Oswestry Disability Index; PRO = patient-reported outcome; $\mathrm{QI}=$ quality improvement; RN = registered nurse.

SUBMITTED July 30, 2015. ACCEPTED October 2, 2015.

INCLUDE WHEN CITING DOI: 10.3171/2015.10.FOCUS15370. 
$\mathrm{O}$ VER the past decade, there have been significant concerns about the utilization of spine surgery in the US. In 2006, the Dartmouth Atlas Project noted that there had been a steadily increasing utilization of lumbar spine surgery, especially lumbar fusion, in the US over the prior decade. ${ }^{24}$ In addition, there was significant variability in surgical practices across the country, with increased utilization being proportional to the number of spine surgeons per capita in the region. Subsequent studies have shown that the long-term outcomes for patients who undergo some operations are worse in areas with high rates of spinal surgery..$^{15}$ In 2011, the Agency for Healthcare Research and Quality found spinal fusions to be the costliest of the 20 most commonly performed operating room procedures in US hospitals. ${ }^{29}$ As a result, there has been increasing pressure on spine surgeons to demonstrate that spinal operations, and especially lumbar spinal operations, provide value.

Despite these findings, a number of prospective studies have illustrated that spinal surgery represents a valuable treatment with long-term clinical outcomes and functional gains that offset the high up-front costs of the initial surgery in appropriately chosen patients. ${ }^{16,17,20,25-28}$ Given the scrutiny of spine surgery by policy makers, spine surgeons are motivated to demonstrate and improve outcomes, by determining which patients will and will not benefit from surgery, and to reduce costs, often by reducing complications. Insurers are similarly motivated. Such concerns have motivated the conception and implementation of the National Neurosurgery Quality and Outcomes Database $\left(\mathrm{N}^{2} \mathrm{QOD}\right){ }^{1,18} \mathrm{~N}^{2} \mathrm{QOD}$ represents a large, national, prospective registry that was first launched in March 2012.2,19 The project is jointly funded by the American Association of Neurological Surgeons and participating sites. A recent article by Asher et al. reported a total enrollment of 7300 patients. ${ }^{3}$ At the time of that report, 2780 patients had crossed the 12-month threshold with a follow-up rate of $81.0 \%$ at 3 months and $74.4 \%$ at 12 months. ${ }^{3}$ Currently, this is the largest society-based, North American, cooperative spine surgery registry.

\section{The BCBSM Value Partnerships Program and CQI Initiatives}

In 2013, Blue Cross Blue Shield of Michigan (BCBSM) and Blue Care Network (BCN) established the Michigan Spine Surgery Improvement Collaborative (MSSIC) as part of their Value Partnerships program. The Value Partnerships program is a collection of clinically oriented initiatives that have significantly improved-and continue to improve-the quality of patient care throughout the state of Michigan. ${ }^{13,21}$ Through Value Partnerships, BCBSM/ $\mathrm{BCN}$ works collaboratively with physicians, physician organizations, and the majority of the acute-care hospitals in the state to improve the health care provided to all Michigan residents. The goals of these collaborations are to enhance clinical quality, decrease complications, manage costs, eliminate errors, and improve health outcomes. This partnership model was the first of its kind nationally and has been regarded as an innovative approach to improving health care quality and value. ${ }^{6,8,21,23}$
As of 2015, BCBSM/BCN funds 22 Collaborative Quality Initiative (CQI) programs (Fig. 1) in both hospitals and physician practice setting. Each initiative includes a coordinating center that works with $\mathrm{BCBSM} / \mathrm{BCN}$, participating hospitals, and physicians to manage data collection and analysis. Physician leaders serve as directors of the coordinating center for each initiative. The coordinating center helps train abstractors at the various hospitals, reviews data quality, and monitors participating sites to ensure data validity. BCBSM/BCN funds participating hospitals and providers to collect, share, and analyze data through the use of clinical registries, similar to other statewide and national registries. ${ }^{1-3,9,19}$ The patients entered into the registries are not restricted to $\mathrm{BCBSM} / \mathrm{BCN}$ subscribers. The registries allow the coordinating centers to develop risk-adjustment models, analyze practice variation, and identify high and low outliers and the "best practices" on a variety of process and outcome measures.

However, while the registries are an essential tool for these collaborative initiatives, they are not the ultimate goal. Measurable, statewide, quality improvement (QI) is the goal. The coordinating center organizes face-to-face meetings 3 to 4 times each year and regular conference calls among participating clinicians in order to identify opportunities to improve quality and implement QI interventions.

This has led to significant improvements in outcomes and reductions in costs for Michiganders undergoing surgery. For example, the Michigan Urological Surgery Improvement Collaborative (MUSIC), a physician practicebased collaborative, has lowered the rate of infection after prostate biopsies across the state. ${ }^{30,31}$ The mechanism for identifying the incidence of this particular complication, identifying the best practice patterns that help minimize this complication, and implementing statewide initiatives all originated within the MUSIC collaborative. The Michigan Arthroplasty Registry Collaborative Quality Initiative has implemented initiatives to reduce transfusions and prevent deep vein thrombosis (DVT), pulmonary embolism, infections, and readmissions.$^{14}$ Other improvements include cost reductions and improvements in quality with the Michigan Surgery Quality Collaborative and the Michigan Breast Oncology Quality Initiative. . $7,10-13,21,22^{2}$

\section{MSSIC Overview}

MSSIC is a statewide, QI collaborative that involves hospitals, orthopedic surgeons, and neurosurgeons. To participate in MSSIC, a hospital must meet 2 eligibility criteria. First, a facility must perform a minimum of 200 spinal procedures annually. Second, a hospital must provide a letter of commitment indicating the willingness of at least 1 neurosurgeon and 1 orthopedic surgeon to act as surgeon champion and be active participants in collaborative activities (e.g., conference calls, quarterly meetings, committees), as well as be local leaders of consortiumwide and site-specific QI initiatives. Each MSSIC hospital therefore has 2 surgeon champions: an orthopedic spine surgeon and a neurosurgeon.

The MSSIC Coordinating Center is housed at the Henry Ford Health System. There are currently 2 codirectors 


\section{CQIs Fit in the Value Partnerships Program}

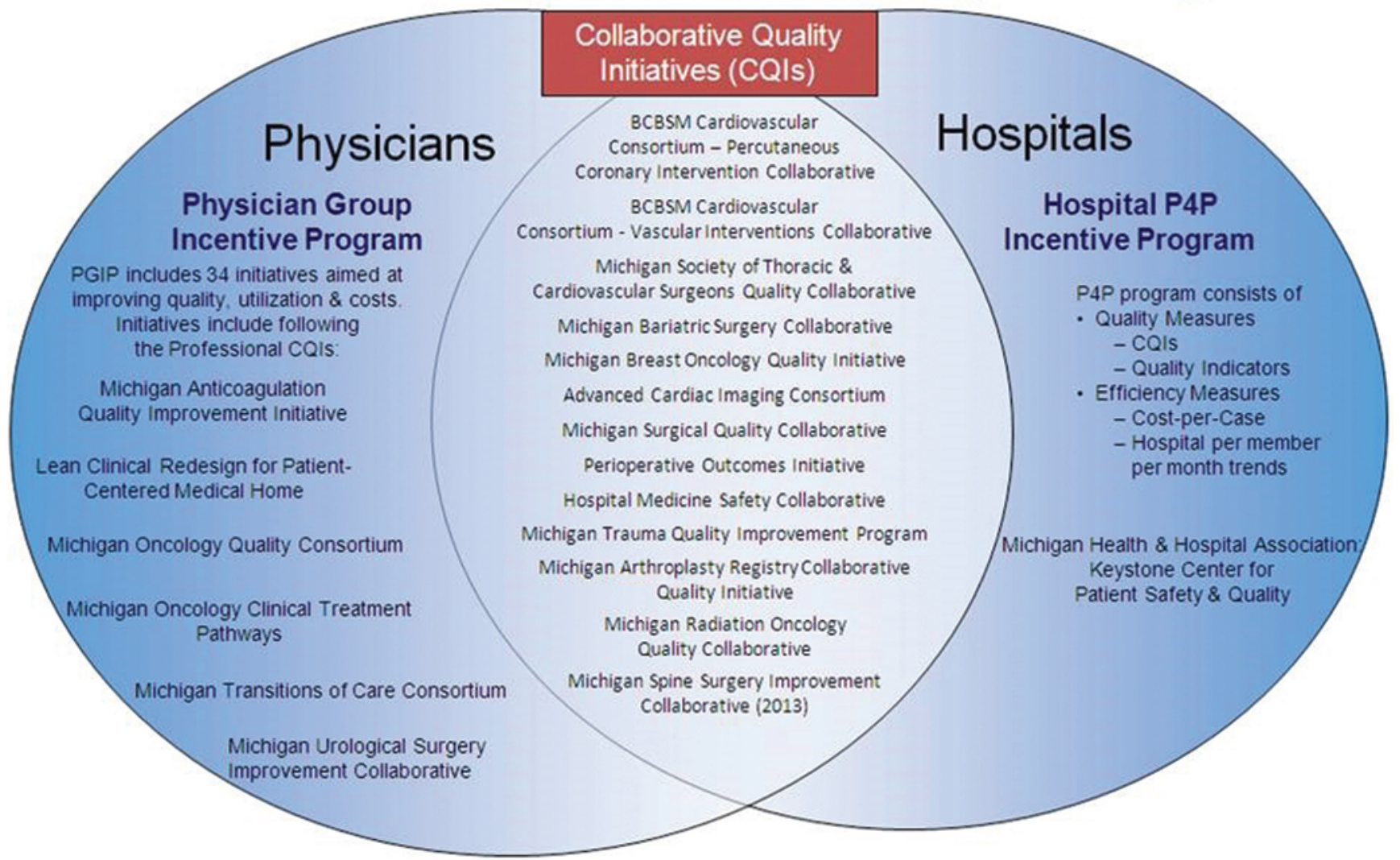

FIG. 1. Illustration of the BCBSM Value Partnership paradigm. In addition to MSSIC, there are 12 other CQIs currently active within the state. PGIP = Physician Group Incentive Program; P4P = pay for performance.

(S.B. and M.A.), 3 associate directors (V.C., J.M.S., and D.R.N.), a program manager (L.P.), a data manager (S.J.), and statisticians (M.J. and N.O.T.), as well as administrative support.

MSSIC focuses on lumbar and cervical spine surgery, specifically indications such as stenosis, disc herniation, and degenerative disease. Surgery for tumors, traumatic fractures, deformity, scoliosis, and acute spinal cord injury are not currently within the scope of MSSIC. Pure thoracic cases are not included in MSSIC. The features of interest include pertinent medical history prior to surgery, surgical indications, details of the surgical procedure, details on immediate postoperative hospitalization, any adverse events or complications within 90 days of surgery, and patient-reported outcomes collected at 90 days, 1 year, and 2 years after surgery.

The patient registry is maintained by ArborMetrix, which also maintains data for some of the other BCBSM CQIs. Participating surgeons have access to their own data in real time, as well as to aggregate, deidentified data from their own and other participating hospitals for benchmarking and comparison purposes. The hospitals and surgeon champions do not have access to identifiable, individual surgeon's data, but they do have access to de-identified, individual-level surgeon data for the purposes of comparison. The registry interface includes custom filters and search functions that allow an individual surgeon to identify meaningful subsets of cases and promote "apples to apples" comparisons with other surgeons and hospitals. Hospitals and surgeons can engage in their own local QI initiatives and track their own data over time. However, they are prohibited from advertising their outcomes relative to other participants. The goal of the collaborative is to "lift all boats."

MSSIC holds quarterly meetings attended by surgeon champions from participating sites, as well as abstractors, quality assurance officers from hospitals, and members of the coordinating center staff. Deidentified data are presented with a letter code for each hospital or surgeon, although centers are free during discussion to disclose which data are theirs. BCBSM/BCN representatives who work with multiple CQIs also sometimes attend, but there is no sharing of data on the performance of hospitals or surgeons with the contracting groups at $\mathrm{BCBSM} / \mathrm{BCN}$. All participants sign nondisclosure agreements about the contents of the meeting, so nothing shared during the meeting leaves the room or is otherwise shared with nonparticipants.

In the early stages of the collaborative, these meetings largely involved examining the quality of the data from the participating centers, training new abstractors, reviewing the literature, and identifying potential quality initiatives. Potential areas of interest include preventing surgi- 
cal complications, reducing the length of hospital stay, assessing the frequency and management of failed back-surgery syndrome, assessing the use of minimally invasive procedures, assessing the use of fusion versus nonfusion procedures, and choosing multilevel versus single-level procedures.

In addition, there are monthly conference calls between the surgeon champions and the coordinating center. There is also an Executive Committee and Abstractor Committee, which were formed to provide general oversight and input from members outside of the coordinating center. The Executive Committee, which is made up of the directors, associate directors, 4 surgeon champions with equal representation by orthopedic and neurosurgeons, and a representative from the Abstractor Committee, meets on a quarterly basis to discuss policy and procedural issues for the collaborative and to authorize publications by participants in the collaborative. The purpose of the Abstractor Committee is to study all aspects of the data collection processes and QI initiatives in order to make recommendations, streamline and improve initiatives, facilitate the exchange of information and ideas, and improve articulation and coordination among all data abstractors. The recommendations are then reviewed by the MSSIC Executive Committee. Once approved, the manual of operations is updated and distributed.

\section{MSSIC Registry Details}

The inclusion criteria for cases in the MSSIC registry include the following spine problems: spondylosis; intervertebral disc disorders; other disorders of the cervical region; other and unspecified disorders of the back; other acquired musculoskeletal deformity (Grade 1 or 2 spondylolisthesis); other congenital musculoskeletal anomalies (Grade 1 or 2 spondylolisthesis); and complications peculiar to certain specified procedures (Fig. 2). Exclusion criteria include: patient age is younger than 18 years; pa- tient is incarcerated; the patient's medical records are unavailable or inaccessible; and cases of moderate $\left(25^{\circ}-50^{\circ}\right)$ and severe $\left(>50^{\circ}\right)$ scoliosis. Additional exclusion criteria include: tumor; meningitis; preexisting spinal infection related to surgery; other disorders of bone and cartilage, spinal deformity, Grades 3 and 4 spondylolisthesis; congenital anomalies of the nervous system; traumatic fracture; and spinal cord injury (Fig. 3).

The data collection process varies between participating sites, depending on the particular workflow of each clinic or hospital, but can be summarized by a general process (Fig. 4). All lumbar and cervical spine surgeries are entered into the MSSIC registry, regardless of whether they are excluded or included for full data entry. Excluded cases are entered to track the volume of excluded cases and document the reasons for exclusion in order to prevent bias in case selection. Once a cervical/lumbar patient is identified in the schedule, the abstractor reviews a "crosswalk" document to see if the patient had been previously entered in the registry. If the patient is not found, then she is considered "new" and added to the MSSIC registry. Once the patient is added, the medical record is reviewed to determine if the patient should be excluded or included for full data entry. Once it is determined that a patient will be included for full data entry, the abstractor continues to collect data from 2 sources: the patient medical record (which consists of the hospital medical record, as well as the surgeon's office record for private practice settings); and patient-reported pain and functional status questionnaires. Baseline patient-reported outcomes (PROs) must be collected within 30 days before surgery.

Confirming the surgery date in the MSSIC registry triggers other time-sensitive milestones for data collection and entry into the registry. Ninety days after the surgery, the abstractor collects data on the surgery that took place and other relevant medical findings. PROs are also collected at 90 days, 1 year, and 2 years after surgery. There

\section{Inclusion Criteria}

- ICD-9 Codes as listed for the following categories:

- Spondylosis and allied disorders

- Intervertebral disc disorders

- Other disorders of cervical region

- Other and unspecified disorders of back

- Other acquired musculoskeletal deformity. (Grades 1 and

2 are included - per radiology report or physician note.)

- Other congenital musculoskeletal anomalies. (Grades 1 and 2 are included - per radiology report or physician note.)

- Complications peculiar to certain specified procedures

- Other (Free text to add new code - For use only with approval of MSSIC Coordinating Center)

- None applicable

FIG. 2. Inclusion criteria by ICD-9 codes for MSSIC. Although pure thoracic cases are excluded, cervical cases that extend into the upper thoracic area, as well as lumbar cases that extend to the lower thoracic area, are still eligible for inclusion. 


\section{Exclusion Criteria}

- Patient is under 18 years of age

- Patient is incarcerated

- Patient medical records or documentation are not available, cannot be accessed

- $\quad$ "Moderate" or "severe" scoliosis as noted, by word or value, on radiology report or consultation note, with or without an ICD-9 Diagnosis Code assigned, is an exclusion criterion. Indicate degree of severity here.

- Moderate (25 to 50 degrees)

- Severe ( $>50$ degrees)

- ICD-9 Codes as listed for the following categories:

○ Tumor (spinal)

○ Meningitis

- Pre-existing spinal infection related to surgery

- Other disorders of bone and cartilage

○ Deformities ("Moderate" and "severe" scoliosis are excluded)

- Spondylolisthesis (Grades 3 and 4 are excluded - per radiology report or physician note.)

- Congenital anomalies of the nervous system (spinal cordrelated)

- Traumatic fracture

- Spinal cord injury

- $\quad$ Other (Free text to add new code - For use only with approval of MSSIC Coordinating Center)

- Review complete (confirming that the case is NOT excluded, therefore is included)

FIG. 3. Exclusion criteria for MSSIC. Traumatic fractures, spinal cord injury, tumors, and significant spinal deformity are exclusion criteria.

are a number of options for collecting these data. The abstractor can obtain the data from the patient directly during an office visit, via a telephone interview, by mailing the questionnaire, or using an online website portal.

The data set for the baseline data consists of general medical history as well as patient-reported pain and functional status measures. The pertinent medical history includes: smoking status, diabetes status, history of coronary artery disease, history of anxiety disorder, history of osteoporosis, history of depression disorder, history of DVT, history of hypercoagulability, current narcotic usage, if the spinal disorder was the result of a motor vehicle injury, workers' compensation claim, employment status, degree of physicality of employment, intention to return to work, ethnicity, and level of education. For patients undergoing lumbar surgery, the indications for surgery are also recorded and include symptomatic lumbar disc herniation, lumbar spondylolisthesis, lumbar stenosis, symptomatic recurrent lumbar disc herniation, lumbar adjacent segment disease, and revision of hardware. In addition, clinical presentation is also recorded, and presenting symptoms include axial pain, radicular pain, muscle weakness, foot drop, and bowel/bladder dysfunction. For patients undergoing cervical surgery, the indications for surgery include disc herniation, foraminal stenosis, central stenosis, cervical instability, cervical adjacent segment disease, and revision of hardware. Presenting symptoms include radicular symptoms with or without neck pain, myelopathy with or without neck or arm pain, and mechanical neck pain from documented cervical instability.

For patient-reported measures, all patients provide the EQ-5D general health state score (0 being worst and 100 being the best health one can imagine) and EQ-5D-3 L (www.euroqol.com). For patients undergoing lumbar surgery, a 0 to 10 numeric rating scale (NRS) for leg and back pain and Oswestry Disability Index (ODI) for back pain are collected. For patients undergoing cervical surgery, a 0 to 10 NRS for arm and neck pain, Neck Disability Index 


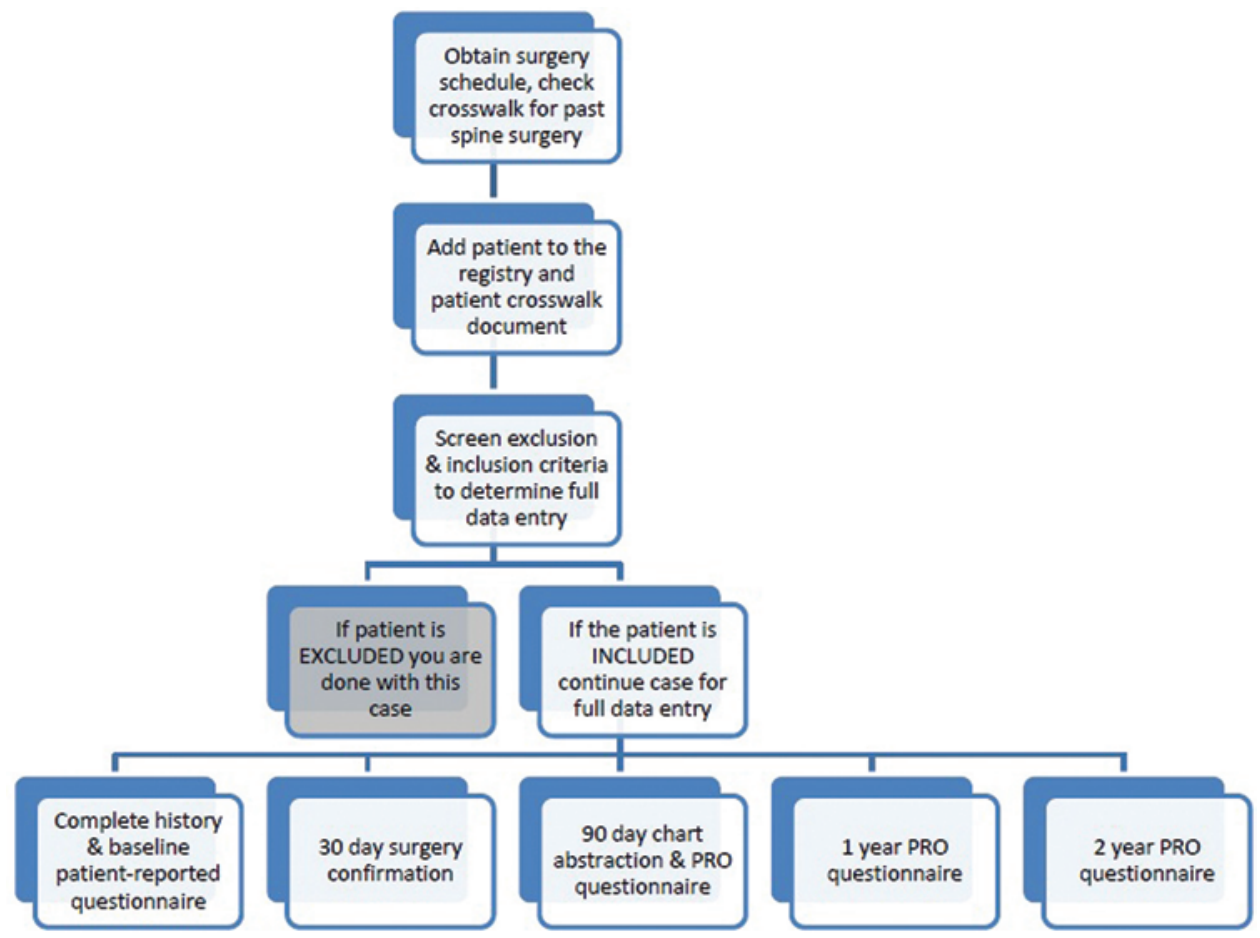

FIG. 4. Illustration of abstraction workflow of patient identification, initial data entry, and subsequent follow-up if the patient meets the inclusion criteria.

(NDI), and modified Japanese Orthopaedic Association (mJOA) score are collected. ${ }^{5}$ The same measures are used at the postsurgery time points, as well as the North American Spine Society Low Back Outcome Instrument. ${ }^{32}$ Options include: "Surgery met my expectations"; "I didn't improve as much as hoped but would undergo the same operation for the same results"; "Surgery helped but I would not undergo the same operation for the same results"; and "I am the same or worse as compared to before surgery."

The surgical details and adverse events are abstracted from the medical records within 90 days of the initial surgery. Surgical variables include: American Society of Anesthesiologists grade; estimated blood loss; surgical time; if the procedure was the second part of a staged operation; if laminectomy/laminotomy/foraminotomy was performed and, if so, how many levels were involved; if arthrodesis was performed; type of arthrodesis techniques; number of segments fused; types of instrumentation (specific to cervical and lumbar surgery); type of bone grafting material; drain placement; use of intraoperative neurophysiological monitoring; and unintentional durotomy. For cervical surgery, the additional variables include: if arthrodesis was performed, if arthrodesis was stopped at C7 or went past the cervicothoracic junction, arthroplasty, and if a corpectomy was performed. Hospital length of stay, ambulation on the first day after surgery, disposition at discharge, use of perioperative antibiotics, and the use of venous thromboembolism prophylaxis were also noted.

Adverse events are also recorded in the registry and obtained primarily from the patient's hospital medical record. Adverse events include DVT, pulmonary embolism, myocardial infarction, urinary tract infection, surgical site infections, urinary retention, postoperative ileus, wound dehiscence, surgical site hematomas requiring reoperation, stroke, new motor deficit, death, any unplanned reoperation, and readmission to hospital within 90 days.

The BCBSM/BCN-sponsored QI collaboratives generally do not have fixed, total patient, accrual targets or planned end dates, and MSSIC is following this pattern. The work of QI never ends, and the project is not designed to test specific scientific hypotheses or answer questions for which a fixed target sample size can be calculated. Since comparative data analysis is done at the level of the individual surgeon and/or hospital, and then at the level of homogeneous patient group (e.g., 2-level lumbar fusions or patients with sciatica as the primary indication for surgery), the overall number of patients in the registry has to be very large to permit this kind of focused analysis. Analyses are done on an ongoing basis, so there is never a point where a target sample size for analysis is reached.

Comparisons in the prospective cohort analyses require complete and unbiased data collection, so site audits focus on the extent to which all eligible cases are included in the registry and the extent to which available data on those cases are entered accurately in the registry.

\section{Evaluation of Participating Hospitals}

Each participating hospital is evaluated each year through a CQI performance index. As a component of the BCBSM/BCN's pay-for-performance incentive program, the performance index (developed by the coordinating center in conjunction with BCBSM) includes a set of measures, and the overall score is shared with BCBSM, which eventually leads to the incentive amount of funding a hospital can earn for participation in MSSIC. In the initial 
years of a hospital collaborative, the measures are related to the quality of participation, such as meeting attendance and the accuracy and timeliness of the data the hospital submits. As the collaborative matures, it is expected that specific measures will be developed related to involvement in QI initiatives, with active participation in such initiatives becoming a more important part of the performance index.

Other expectations for participating hospitals include identifying or hiring key personnel. Each hospital has an administrative lead who is the administrative contact for MSSIC at that facility. This person provides institutional support for full-project participation and is expected to attend collaborative meetings. The facility is also expected to identify or hire 1 or 2 data abstractors. A single fulltime employee (FTE) is expected to effectively manage 376 eligible cases, and the facility is expected to staff the MSSIC project accordingly. The abstractors should be a registered nurse (RN), registered health information technician, research coordinator, or other trained health care professional. The abstractors are responsible for timely and accurate collection of data and the entry of data into a web-based data form. In addition, the abstractors are expected to attend all collaborative meetings and conference calls. Finally the abstractors are expected to participate in coordinating center-led audits of the charts of patients who were entered into the MSSIC registry in order to ensure quality data collection.

Funding for data abstractors is provided by BCBSM/ $\mathrm{BCN}$. Participating hospitals receive annual funding from BCBSM/BCN based on their case volume. This payment is intended to cover the portion of the data abstraction costs for BCBSM and BCN plans, government-paid (Medicare and Medicaid) cases, and uninsured cases (projected to be approximately $80 \%$ of total cases). The FTE amount is based on $80 \%$ of a fully loaded RN salary, as determined by BCBSM with input from the coordinating centers. BCBSM/BCN support will cover an amount equal or up to $80 \%$ of 2 full-time RNs (1.6 FTEs) at a participating site. If the case volume exceeds the estimated workload of 2 FTEs, then a sampling strategy is put into place so that the number of cases matches the capability of 2 dedicated FTEs. The sampling strategy is developed by the participating site and the coordinating center's statistician. Alternatively, the participating site maintains the right to hire additional abstractors beyond the 2 FTEs funded by $\mathrm{BCBSM} / \mathrm{BCN}$, if it so chooses.

$\mathrm{BCBSM} / \mathrm{BCN}$ supports MSSIC (the coordinating center and the participating hospitals or group practices) through annual contracts, with no planned or fixed end point to the collaborative. MSSIC is 1 of 21 other statewide CQIs sponsored by BCBSM/BCN, and 1 collaborative has been in existence since mid-1997. ${ }^{6,8,13,14,21}$ The value of the CQIs to BCBSM and BCN, BCBSM and BCN members, and Michigan in general has been sufficiently well established to support annual funding, as long as the collaboratives continue to be successful. Three collaboratives retired after fulfilling their full potential or were unsustainable in their current state. The clearly stated goal is statewide QI, not the identification or creation of "centers of excellence." If individual surgeons or hospitals are identified as doing particularly well in a specific domain (e.g., PROs, prevention of complications), they are asked to share their practices and protocols with others. Analysis of data from the registry may identify either particularly effective or ineffective procedures; it is expected that the members of the collaborative will shift to the former and away from the latter, without any need for a decision about reimbursement from BCBSM/BCN. As mentioned, BCBSM/BCN does not see identifiable data. CQIs promote partnerships with physicians, physician groups, and hospitals to create strong collaboration and reward systems for the transformation of health care.

Even though MSSIC is a QI initiative and not research, each site participating in MSSIC must obtain either approval or exemption from its local institutional review board (IRB) prior to actively participating in MSSIC. While all IRBs are different, in most cases the IRB concludes that MSSIC is not research as defined in 45 CFR 46.102(d) and is exempt from IRB review. If the IRB does identify the activity as subject to IRB review and approval, the MSSIC project would most likely be reviewed through the expedited procedure and considered no more than minimal risk to the subject based on 45 CFR 46.110 of the US Department of Health \& Human Services Code of Federal Regulations (CFR). If the project is considered to be research at a particular hospital, that IRB generally rules that the criteria for waiver of patient consent have been met, in accordance with the Health Insurance Portability and Accountability Act, Privacy Rule, Section 164.512 (i) (http:// www.gpo.gov/fdsys/pkg/CFR-2011-title45-vol1/pdf/CFR2011-title45-vol1-sec164-512.pdf).

With respect to other larger, national programs from the initial planning discussions, the MSSIC registry and its contents were designed to be compatible with the $\mathrm{N}^{2} \mathrm{QOD}$ registry in order to allow direct comparisons between the 2 and permit the uploading of data (if mutually agreed) from MSSIC to $\mathrm{N}^{2} \mathrm{QOD}$. Some minor changes have been made to maximize participation and data completeness in MSSIC, but, for the most part, the registry contents and opportunities for analysis are comparable between $\mathrm{N}^{2} \mathrm{QOD}$ and MSSIC.

\section{Progress to Date}

The initial phase of live data collection began in the spring of 2014 and consisted of the 7 Wave 1 hospitals (Fig. 5). The second phase of recruitment involved 15 additional institutions across the state, which began to enroll patients during the first half of 2015 (Fig. 5). A third phase of enrollment is planned for 2016. According to the most current data, there are 32 hospitals within the state of Michigan that meet the eligibility criteria to be included in MSSIC.

As of July 1, 2015, a total of 6397 cases have been entered into the registry (Fig. 6). Of these 6397 cases, 1573 cases were excluded: 534 cases are pending confirmation of surgery; 269 cases had cancelled surgery; 51 cases are under review; and 719 were excluded following analyses of the exclusion and inclusion criteria. Therefore, 4824 total cases were eligible for analysis. Of these 4824 eligible cases, 1486 cases are still in the 120-day abstraction window range with incomplete data. This leaves 3338 confirmed 


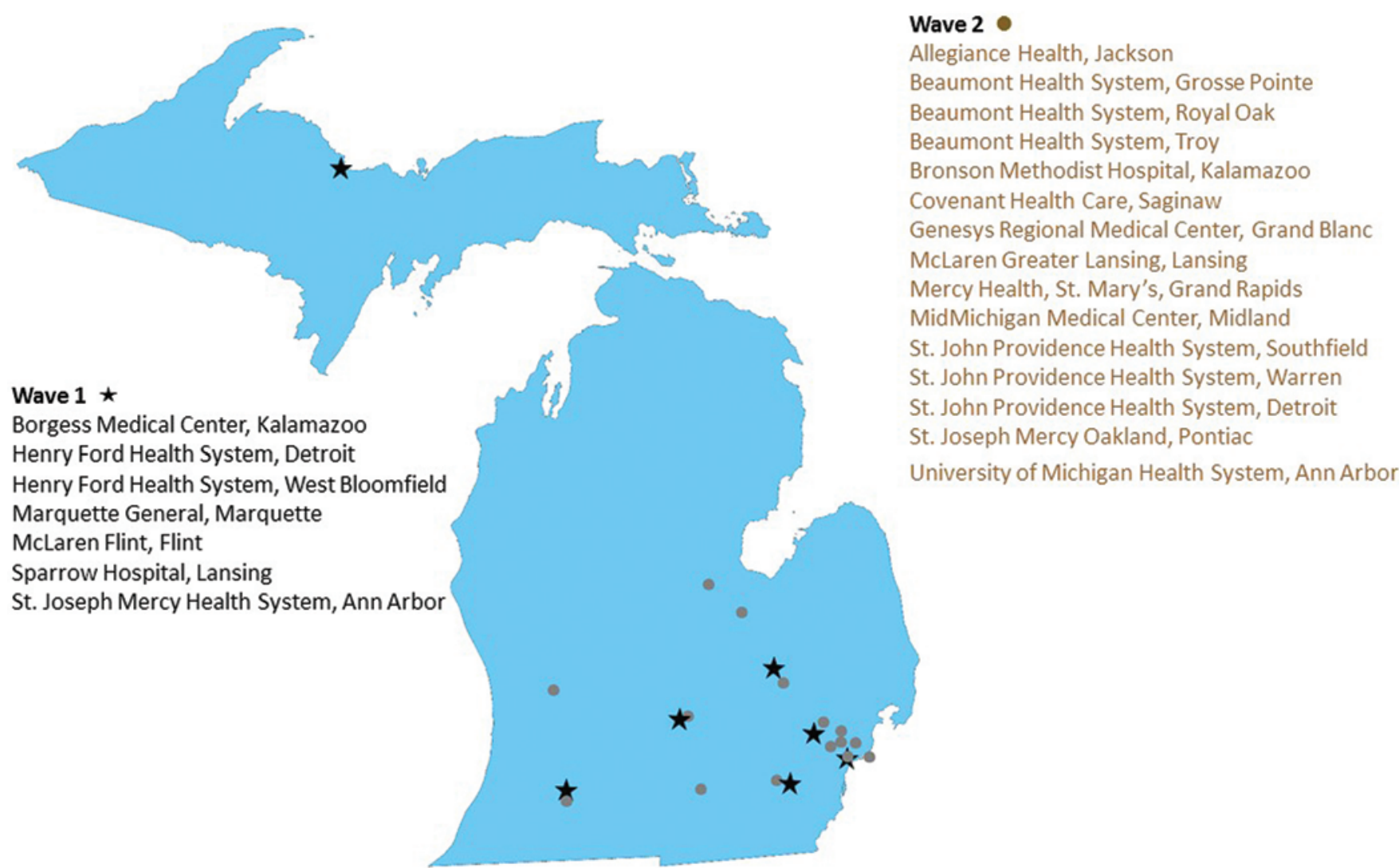

FIG. 5. Current enrollment of MSSIC across the state of Michigan. Currently 22 of 32 total hospitals that are eligible to join MSSIC (centers with at least 200 spinal operations annually) are active participants, with a third wave of recruitment to begin in 2016.

cases with completed abstraction for at least the baseline and 90-day follow-up time points. The distribution of cases is illustrated in Fig. 6. Among these 3338 patients, there are a total of 2469 lumbar cases, 862 cervical cases, and 7 combined procedures. Of the 2469 lumbar cases, 890 are instrumented cases, 1505 are noninstrumented, and 74 are unclassified regarding instrumentation. Of the 862 cervical cases, 696 are instrumented, 132 are noninstrumented, and 34 are unclassified for instrumentation.

\section{Data Collection Efficiency}

Data collection in MSSIC has been active for just over a year in 5 hospitals; most hospitals that are currently active in MSSIC joined in 2015 and are just beginning to establish a base rate of data completeness. None of the hospitals have been active for long enough to have a body of data at 12 months postsurgery, but the first 5 hospitals have enough data to establish preliminary rates of performance for baseline and 90-day data collection. In general, the medical record abstraction variables are obtained with high levels of completeness; the baseline and follow-up patient surveys have been a challenge and are the subject of active-process improvement efforts.

For baseline patient-history abstraction, the completion rates (all information about a case available to an abstractor that has been entered into the registry) range from $93.7 \%$ to $99.6 \%$ across all categories. For surgical details, completeness ranged from $98.9 \%$ to $99.7 \%$ across all pertinent surgical variables. For 90 -day morbidity data, abstraction completion rates range from $97.3 \%$ to $99.3 \%$ across all variables considered.

For the patient surveys, the primary measure of completeness is "percent of eligible patients with an analyzable survey score." This measure excludes any patient surveys that are incomplete or otherwise unscorable. By this measure, the baseline patient survey rate for lumbar patients is $70.7 \%$ (range $54 \%-83 \%$ by hospital) and $70.9 \%$ for cervical patients (range 34\%-90\% by hospital). (Generally, a patient who completed 1 specific measure would complete all, so completion rates did not vary significantly from survey to survey.) At the 90-day follow-up period, the completion rate for lumbar patients is $72.7 \%$ (range $58 \%-81.6 \%$ by hospital) and $72.5 \%$ for cervical patients (range $38.5 \%-97.9 \%$ by hospital).

\section{Data Auditing}

We monitor data completeness for variables abstracted from medical records in 2 ways. One is the percentage of eligible cases for which the abstractor has made some entry in the registry for each variable. By that measure, the completeness of the abstracted variables in the first 5 hospitals during the 1st year was close to $100 \%$. If information for a variable is not available in the medical records or other data source, an abstractor has the option of entering "not documented" or "unknown." The second measure of completeness is the proportion of eligible cases for which 


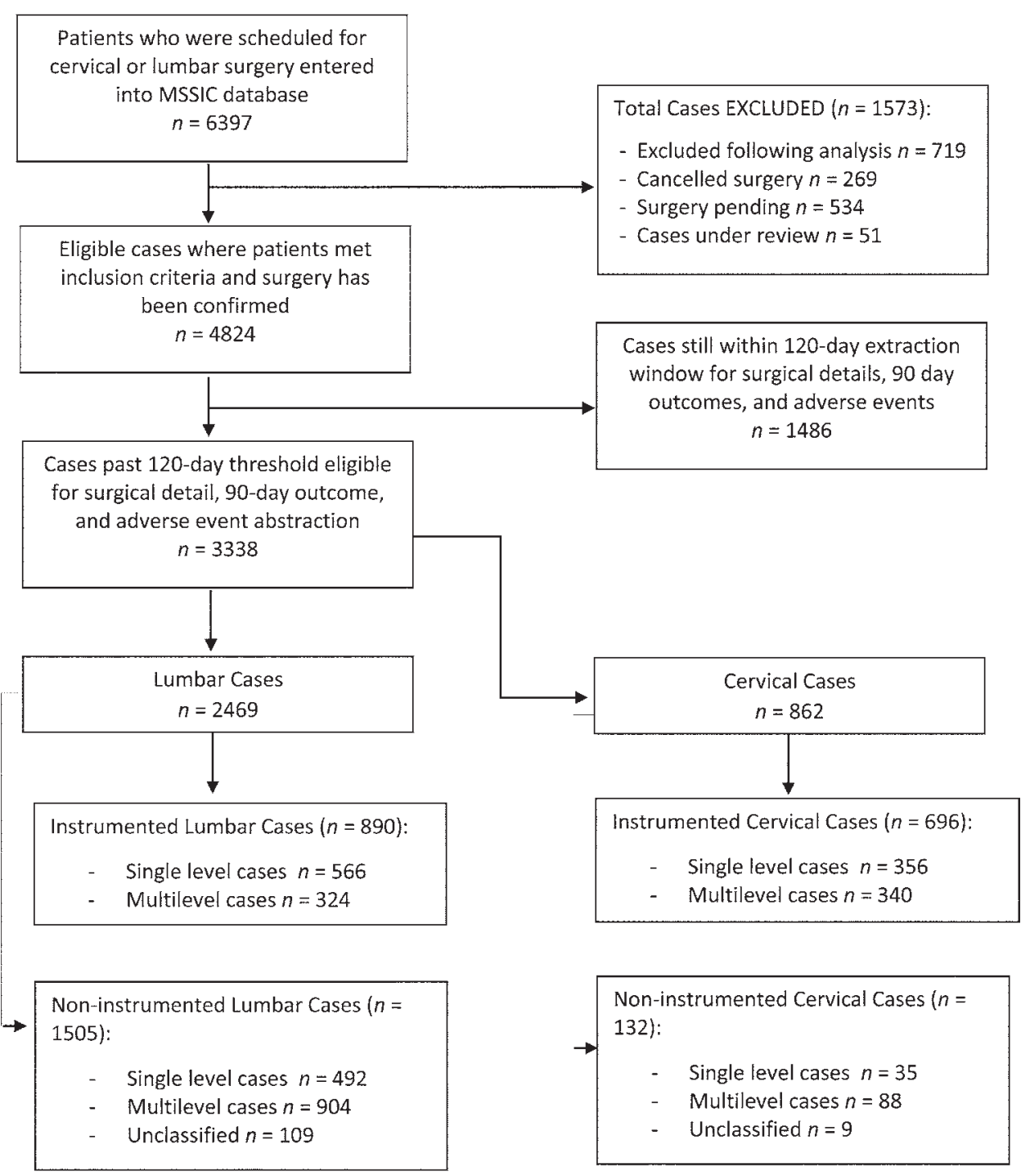

FIG. 6. Illustration of data sources and current progress as of July 1, 2015.

an "analyzable" response option is entered (e.g., "yes" or "no"), but not "not documented." By this second measure of completeness for abstracted information, the rates have ranged from $71 \%$ to $96 \%$. Abstractor and surgeon work groups are continually engaged in working to increase these rates.

The rates of patient survey completeness are not acceptable for the long term, and we are working actively to improve them. In 2 of the 5 hospitals (those with the lowest rates), challenges in recruiting and retaining abstractors led to a significant number of patients who were not asked to complete surveys; we believe this problem has largely been solved. Beyond that, the contractual structure of the project may have contributed to the initially low survey completion rates. Patient contacts are generally made by abstractors who are hospital employees since BCBSM's financial support for participation in MSSIC goes to hospitals, not surgeon practices. Patients are typically seen in surgeon practices before surgery, though, and then again in follow-up after surgery, rather than in the hospital. We are actively working to enhance coordination between hospital abstractors, surgeons' office staff, and the first 5 hospitals and establish smooth processes at the new hospitals in order to achieve a target completion rate of at least $80 \%$.

\section{Diagnostic Distribution}

A summary of these distributions can be found in Table 1. For lumbar cases, the distribution of the diagnoses is as follows: lumbar stenosis, $69.0 \%$; lumbar disc herniation, 47.9\%; lumbar spondylosis, 24.8\%; recurrent lumbar disc herniation, 5.6\%; lumbar adjacent-segment disease, 2.6\%; and lumbar revision of hardware, $2.7 \%$. The categories are not mutually exclusive, and multiple indications are possible for an individual patient. For cervical cases, the distribution of diagnoses is as follows: cervical foraminal stenosis, 56.1\%; cervical central stenosis, $46.4 \%$; cervical disc herniation, $44.7 \%$; cervical instability, $6.8 \%$; cervical adjacent segment disease, $3.1 \%$; and cervical revision of hardware, $2.0 \%$. As with lumbar cases, the cervical indications for surgery are also not mutually exclusive. 
TABLE 1. Diagnostic distribution*

\begin{tabular}{clc}
\hline Spine Region & Indication for Surgery & Percentage of Cases \\
\hline Lumbar & Disc herniation & $47.9 \%$ \\
\hline & Spondylolisthesis & $24.8 \%$ \\
\hline & Stenosis & $69.0 \%$ \\
\hline & Recurrent disc herniation & $5.6 \%$ \\
\hline & Adjacent-segment disease & $2.6 \%$ \\
\hline \multirow{2}{*}{ Cervical } & Hardware revision & $2.7 \%$ \\
\hline & Disc herniation & $44.7 \%$ \\
\hline & Foraminal stenosis & $56.1 \%$ \\
\hline & Central stenosis & $46.4 \%$ \\
\hline & Instability & $6.8 \%$ \\
\hline & Adjacent-segment disease & $3.1 \%$ \\
\hline & Hardware revision & $2 \%$ \\
\hline
\end{tabular}

${ }^{*}$ Cases entered into MSSIC can have multiple indications.

\section{Preliminary Outcome Findings}

As expected, the rates of most postsurgical complications at the 90-day follow-up point have been low. For example, to date the rate for pulmonary embolism is $0.85 \%$, wound dehiscence is $1.11 \%$, and death is $0.29 \%$, while hospital readmission rates have been approximately $8.49 \%$ to date.

Our analysis of PROs at 90 days after surgery focused on the percentage of patients achieving the minimum clinically important difference (MCID) in each of the measured domains. For example, in analyses done for our June 2015 quarterly meeting, among patients with single-level lumbar fusion procedures, the percentage that achieved MCID in the 0 to 10 rating of leg pain ranged from $60 \%$ to 91\% among those hospitals with more than 5 cases available for analysis, and the percentage that achieved MCID on the ODI ranged from $32 \%$ to $80 \%$ in the same hospitals. Among the patients who underwent cervical fusion procedures, the percentage who achieved MCID in the 0 to 10 rating of arm pain ranged from $25 \%$ to $60 \%$ among those hospitals with more than 5 cases available for analysis, and the percentage that achieved MCID on the NDI ranged from $35 \%$ to $60 \%$. In terms of patient satisfaction, $63 \%$ of patients felt that their surgery met expectations and $22 \%$ of patients felt their improvement was short of expectations but would still be willing to undergo the same operation for the same results.

\section{Conclusions}

Prospective registries represent a means of data collection to help improve quality and possibly define "value." Large society-based registries such as $\mathrm{N}^{2} \mathrm{QOD}$ represent a monumental step forward in efforts to define the value of spine surgery. $\mathrm{N}^{2} \mathrm{QOD}$ represents one of many similar efforts worldwide. ${ }^{18}$ There is a wide range of registries, some which have great granularity, such as the Vanderbilt Prospective Spine Registry. Other registries deal with a narrower range of indications or surgical techniques (e.g., Society for Minimally Invasive Spine Surgery, Chest Wall and Spine Deformity), while still others are large national or multinational registries with over 70,000 patients (e.g., SweSpine, SpineTango).

MSSIC represents a regional (State of Michigan) QI initiative that uses a registry as one of its tools for QI, encompassing a comprehensive range of cervical and lumbar surgery cases for the most common degenerative conditions at both academic and nonacademic centers in both rural and urban settings. MSSIC is funded by a large private payer (BCBSM/BCN) and one of several collaboratives within the state of Michigan that is funded by BCBSM/ BCN. MSSIC will be able to identify potential areas of QI in spine surgery and provide a vehicle from which to convene and coordinate local and potentially statewide quality initiatives across all participating hospitals. Our goals are to improve patient safety and to lower costs.

To date, MSSIC has been successfully implemented in 7 Phase 1 hospitals and has been actively accruing data for over a year at the time of this writing. The current total of 4824 analyzable cases reflects primarily the contribution of these 7 institutions alone. With the recent addition of 15 Phase 2 hospitals, the rate of recruitment is expected to accelerate dramatically with a projected annual recruitment of over 10,000 cases per year. Although MSSIC is limited in being a regional registry for a midsized state, MSSIC does have the potential for gleaning valuable insights from a large volume of lumbar and cervical spine surgeries across urban and rural settings and academic and nonacademic practices.

\section{Acknowledgments}

BCBSM disclaimer statement: Although BCBSM and MSSIC work collaboratively, the opinions, beliefs, and viewpoints expressed by the authors do not necessarily reflect the opinions, beliefs, and viewpoints of BCBSM or any of its employees.

BCBMS support statement: Support for MSSIC is provided by BCBSM and BCN as part of the BCBSM Value Partnerships program.

\section{References}

1. Asher AL, Devin CJ, Mroz T, Fehlings M, Parker SL, McGirt MJ: Clinical registries and evidence-based care pathways: raising the bar for meaningful measurement and delivery of value-based care. Spine (Phila Pa 1976) 39 (22 Suppl 1):S136-S138, 2014

2. Asher AL, McCormick PC, Selden NR, Ghogawala Z, McGirt MJ: The National Neurosurgery Quality and Outcomes Database and NeuroPoint Alliance: rationale, development, and implementation. Neurosurg Focus 34(1):E2, 2013

3. Asher AL, Speroff T, Dittus RS, Parker SL, Davies JM, Selden N, et al: The National Neurosurgery Quality and Outcomes Database (N2QOD): a collaborative North American outcomes registry to advance value-based spine care. Spine (Phila Pa 1976) 39 (22 Suppl 1):S106-S116, 2014

4. Barnes GD, Birkmeyer N, Flanders SA, Froehlich JB, Hemmila M, Henke PK, et al: Venous thromboembolism: a collaborative quality improvement model for practitioners, hospitals, and insurers. J Thromb Thrombolysis 33:274-279, 2012

5. Benzel EC, Lancon J, Kesterson L, Hadden T: Cervical laminectomy and dentate ligament section for cervical spondylotic myelopathy. J Spinal Disord 4:286-295, 1991

6. Birkmeyer NJ, Birkmeyer JD: Strategies for improving surgical quality - should payers reward excellence or effort? $\mathbf{N}$ Engl J Med 354:864-870, 2006 
7. Birkmeyer NJ, Dimick JB, Share D, Hawasli A, English WJ, Genaw J, et al: Hospital complication rates with bariatric surgery in Michigan. JAMA 304:435-442, 2010

8. Birkmeyer NJ, Share D, Campbell DA Jr, Prager RL, Moscucci M, Birkmeyer JD: Partnering with payers to improve surgical quality: the Michigan plan. Surgery 138:815-820, 2005

9. Breakwell LM, Cole AA, Birch N, Heywood C: Should we all go to the PROM? The first two years of the British Spine Registry. Bone Joint J 97-B:871-874, 2015

10. Breslin TM, Caughran J, Pettinga J, Wesen C, Mehringer A, Yin $\mathrm{H}$, et al: Improving breast cancer care through a regional quality collaborative. Surgery 150:635-642, 2011

11. Campbell DA Jr: Quality improvement is local. J Am Coll Surg 209:141-143, 2009

12. Campbell DA Jr, Englesbe MJ, Kubus JJ, Phillips LR, Shanley CJ, Velanovich V, et al: Accelerating the pace of surgical quality improvement: the power of hospital collaboration. Arch Surg 145:985-991, 2010

13. Englesbe MJ, Dimick JB, Sonnenday CJ, Share DA, Campbell DA Jr: The Michigan surgical quality collaborative: will a statewide quality improvement initiative pay for itself? Ann Surg 246:1100-1103, 2007

14. Hughes RE, Hallstrom BR, Cowen ME, Igrisan RM, Singal BM, Share DA: Michigan Arthroplasty Registry Collaborative Quality Initiative (MARCQI) as a model for regional registries in the United States. Orthop Res Rev 7:47-56, 2015

15. Keller RB, Atlas SJ, Soule DN, Singer DE, Deyo RA: Relationship between rates and outcomes of operative treatment for lumbar disc herniation and spinal stenosis. J Bone Joint Surg Am 81:752-762, 1999

16. Lurie JD, Tosteson TD, Tosteson A, Abdu WA, Zhao W, Morgan TS, et al: Long-term outcomes of lumbar spinal stenosis: eight-year results of the Spine Patient Outcomes Research Trial (SPORT). Spine (Phila Pa 1976) 40:63-76, 2015

17. Lurie JD, Tosteson TD, Tosteson AN, Zhao W, Morgan TS, Abdu WA, et al: Surgical versus nonoperative treatment for lumbar disc herniation: eight-year results for the spine patient outcomes research trial. Spine (Phila Pa 1976) 39:3-16, 2014

18. McGirt MJ, Parker SL, Asher AL, Norvell D, Sherry N, Devin CJ: Role of prospective registries in defining the value and effectiveness of spine care. Spine (Phila Pa 1976) 39 (22 Suppl 1):S117-S128, 2014

19. McGirt MJ, Speroff T, Dittus RS, Harrell FE Jr, Asher AL: The National Neurosurgery Quality and Outcomes Database (N2QOD): general overview and pilot-year project description. Neurosurg Focus 34(1):E6, 2013

20. Park DK, An HS, Lurie JD, Zhao W, Tosteson A, Tosteson TD, et al: Does multilevel lumbar stenosis lead to poorer outcomes?: a subanalysis of the Spine Patient Outcomes Research Trial (SPORT) lumbar stenosis study. Spine (Phila Pa 1976) 35:439-446, 2010

21. Share DA, Campbell DA, Birkmeyer N, Prager RL, Gurm HS, Moscucci M, et al: How a regional collaborative of hospitals and physicians in Michigan cut costs and improved the quality of care. Health Aff (Millwood) 30:636-645, 2011

22. Smith M, Hussain A, Xiao J, Scheidler W, Reddy H, Olugbade $\mathrm{K} \mathrm{Jr}$, et al: The importance of improving the quality of emergency surgery for a regional quality collaborative. Ann Surg 257:596-602, 2013

23. Waljee JF, Birkmeyer NJ: Collaborative quality improvement in surgery. Hand Clin 30:335-343, vi, 2014

24. Weinstein JN, Lurie JD, Olson PR, Bronner KK, Fisher ES:
United States' trends and regional variations in lumbar spine surgery: 1992-2003. Spine (Phila Pa 1976) 31:2707-2714, 2006

25. Weinstein JN, Lurie JD, Tosteson TD, Skinner JS, Hanscom $\mathrm{B}$, Tosteson AN, et al: Surgical vs nonoperative treatment for lumbar disk herniation: the Spine Patient Outcomes Research Trial (SPORT) observational cohort. JAMA 296:2451-2459, 2006

26. Weinstein JN, Lurie JD, Tosteson TD, Tosteson AN, Blood EA, Abdu WA, et al: Surgical versus nonoperative treatment for lumbar disc herniation: four-year results for the Spine Patient Outcomes Research Trial (SPORT). Spine (Phila Pa 1976) 33:2789-2800, 2008

27. Weinstein JN, Lurie JD, Tosteson TD, Zhao W, Blood EA, Tosteson AN, et al: Surgical compared with nonoperative treatment for lumbar degenerative spondylolisthesis. fouryear results in the Spine Patient Outcomes Research Trial (SPORT) randomized and observational cohorts. J Bone Joint Surg Am 91:1295-1304, 2009

28. Weinstein JN, Tosteson TD, Lurie JD, Tosteson A, Blood E, Herkowitz H, et al: Surgical versus nonoperative treatment for lumbar spinal stenosis four-year results of the Spine Patient Outcomes Research Trial. Spine (Phila Pa 1976) 35:1329-1338, 2010

29. Weiss AJ, Elixhauser A, Andrews RM: Characteristics of operating room procedures in US Hospitals, 2011. Healthcare Cost and Utilization Project Statistical Brief 170. Rockville, MD: Agency for Healthcare Research and Quality, 2011 (https://www.hcup-us.ahrq.gov/reports/statbriefs/ sb170-Operating-Room-Procedures-United-States-2011.jsp) [Accessed October 9, 2015]

30. Womble PR, Dixon MW, Linsell SM, Ye Z, Montie JE, Lane $\mathrm{BR}$, et al: Infection related hospitalizations after prostate biopsy in a statewide quality improvement collaborative. J Urol 191:1787-1792, 2014

31. Womble PR, Linsell SM, Gao Y, Ye Z, Montie JE, Gandhi TN, et al: A statewide intervention to reduce hospitalizations after prostate biopsy. J Urol 194:403-409, 2015

32. Wood EG III, Hanley EN Jr: Lumbar disc herniation and open limited discectomy: indications, techniques, and results. Oper Tech Orthop 1:23-28, 1991

\section{Disclosures}

Dr. Chang is a consultant for Globus. Dr. Bartol owns stock and patents with Sentio. Dr. Abdulhak is a consultant for Interga and DePuy Synthes and owns stock in Innovative Surgical Solutions.

\section{Author Contributions}

Conception and design: Schwalb, Bartol, Abdulhak, Chang. Acquisition of data: Pietrantoni, Schwalb, Nerenz, Jones, Jankowski, Oja-Tebbe, Abdulhak. Analysis and interpretation of data: Chang, Schwalb, Nerenz, Bartol, Abdulhak. Drafting the article: Chang, Nerenz. Reviewed submitted version of manuscript: Chang. Approved the final version of the manuscript on behalf of all authors: Chang. Statistical analysis: Nerenz. Study supervision: Schwalb, Nerenz, Abdulhak.

\section{Correspondence}

Victor Chang, Department of Neurosurgery, Henry Ford West Bloomfield Hospital, 6777 W. Maple Blvd., West Bloomfield, MI 48322. email: vchang1@hfhs.org. 